\title{
Flexural vibration band gap and stress concentration properties of periodic beam with functionally graded materials (withdrawal notice)
}

Jihong Wen, Dianlong Yu, Yong Xiao, Mingyao Yang

Jihong Wen, Dianlong Yu, Yong Xiao, Mingyao Yang, "Flexural vibration band gap and stress concentration properties of periodic beam with functionally graded materials (withdrawal notice)," Proc. SPIE 9064, Health Monitoring of Structural and Biological Systems 2014, $90641 Z$ (9 March 2014); doi: $10.1117 / 12.2046093$

EDIE $\quad$ Event: SPIE Smart Structures and Materials + Nondestructive Evaluation and Health Monitoring, 2014, San Diego, California, United States 


\section{Flexural vibration band gap and stress concentration properties of periodic beam with functionally graded materials (withdrawal notice)}

Proc. SPIE 9064, 906420 (2014); http://dx.doi.org/ 10.1117/ 12.2046093

Online Publication Date: 9 March 2014

Withdrawn from Publication: 15 May 2014

Conference Date: Sunday 10-13 March 2014

Conference Location: San Diego, California, United States

Conference Title:

Health Monitoring of Structural and Biological Systems 2014

Conference Chairs: Tribikram Kundu

Jihong Wen, Dianlong, Yong Xiao, and Mingyao Yang

National Univ. of Defense Technology (China)

This paper has been withdrawn by th e publisher because it was not presented at the conference. 